\section{“Health consequences associated with frequent wheezing in adolescents without asthma diagnosis." K. Yeatts, K. Johnston Davis, D. Peden and C. Shy. Eur Respir J 2003; 22: 781-786.}

Statement from the editor:

In November 2003, the European Respiratory Journal published an article entitled "Health consequences associated with frequent wheezing in adolescents without asthma diagnosis" [1]. It was subsequently brought to our attention that another article by two of the same authors, "Health consequences for children with undiagnosed asthma-like symptoms," had been published in the Archives of Pediatric and Adolescent Medicine (now JAMA Pediatrics) [2]; the National Library of Medicine has decided that these two articles represent duplicate publication, and both articles have been labelled as duplicate publications in MEDLINE. A corresponding "notice of duplicate publication" has also been published by the Archives of Pediatric and Adolescent Medicine [3].

Statement from the author:

We are responding to concerns regarding duplicate publication of our analyses and conclusions in the European Respiratory Journal and Archives of Pediatric and Adolescent Medicine. We would first like to state that we had no intention of submitting the same article to different journals, and in this letter we present our perspective on how these two published articles are different.

The APAM publication, "Health consequences for children with undiagnosed asthma-like symptoms," presents the prevalence and functional and health consequences of asthma-like symptoms in the entire study population of 128568 schoolchildren [1]. The ERJ publication, "Health consequences associated with frequent wheezing in adolescents without asthma diagnosis," does not use the entire study population of 128568 as its denominator, but rather focuses on the prevalence and functional and health consequences of children with frequent wheezing but with no diagnosis of asthma ( $\mathrm{n}=7587)$ [2].

We wanted to draw particular attention to this group of children with frequent wheezing and no diagnosis; these children experience functional and health consequences equal to and even greater than children with physician-diagnosed asthma. The ERJ publication demonstrates that the undiagnosed frequent wheezers experience emergency room visits and hospitalisations to an almost equal extent to those of diagnosed asthmatics, yet have significantly fewer physician visits. This conclusion cannot be drawn from the APAM publication.

The APAM publication draws attention to the finding that current wheezing (28\%) in this population of children was quite common (almost one in three). The APAM article is intended for the practising US paediatrician or family doctor; the ERJ article is intended for respiratory specialists and research audience. We were surprised with the allegation of duplicate publication and all the authors sincerely regret any appearance of duplication in these two publications. We wish to reiterate that these papers each provide distinct findings and implications for practising clinicians and paediatric asthma researchers.

\section{Karin Yeatts}

Dept of Epidemiology, Gillings School of Global Public Health, University of North Carolina at Chapel Hill, Chapel Hill, NC, USA.

\section{References}

1 Yeatts K, Johnston Davis K, Peden D, et al. Health consequences associated with frequent wheezing in adolescents without asthma diagnosis. Eur Respir J 2003; 22:781-786.

2 Yeatts K, Shy C, Sotir M, et al. Health consequences for children with undiagnosed asthma-like symptoms. Arch Pediatr Adolesc Med 2003; 157: 540-544.

3 Notice of duplicate publication. Arch Pediatr Adolesc Med 2004; 158: 627.

Eur Respir J 2015; 46: 1532 | DOI: 10.1183/09031936.03.50095803 